\title{
The evaluation of cerebral venous normal anatomy and variations by phase-contrast cranial magnetic resonance venography
}

\author{
E. Doğan¹, M. Apaydın² \\ 'Department of Radiology, Faculty of Medicine, Muğla Sıtkı Koçman University, Muğla, Turkey \\ ${ }^{2}$ Department of Radiology, Izmir Atatürk Education and Research Hospital, Izmir, Turkey
}

[Received: 5 January 2020; Accepted: 5 February 2021; Early publication date: 1 March 2021]

Background: The aim of our study was to determine the ability of the phase-contrast-cranial magnetic resonance venography (PC-CMRV) technique to detect cranial anatomy, variations, thrombosis, to reveal the deficits of the technique and to discuss the reasons for these deficits on a physics basis.

Materials and methods: Phase-contrast's detection rates of anatomic variations and physiological filling defects (FDs) were evaluated in 136 patients and compared with the time-of-flight technique magnetic resonance imaging (MRI) and cadaveric studies.

Results: The dominance correlation between the three evaluated sinuses (transverse sinus [TS], sigmoid sinus, jugular vein) which originated from different embryological buds was statistically significant and the right vessel chain was dominant. $P C$ is inadequate to show some vessels like inferior sagittal sinus (anatomically, this vessel is approximately present in $100 \%$ of the cases, but it was only visualised in $41.2 \%$ of the patients in PC-MRI). Visualisation of major veins was sufficient. PC-MRI created physiological FDs in $27.2 \%(72.3 \%$ middle, $10.3 \%$ inner, $17 \%$ outer part) of the patients. The FDs were concentrated in the middle part and not observed in the dominant sinus.

Conclusions: The defects of visualisation are present due to the $P C$ 's technique. It can be misdiagnosed as agenesis or thrombosis. PC creates a high incidence of physiologic FDs in TS. The results are not reliable, especially if FDs are in the middle part or non-dominant side. (Folia Morphol 2022; 81, 2: 314-323)

Key words: magnetic resonance, venography, dural sinuses, phase contrast, arachnoid granulations

\section{INTRODUCTION}

Cranial magnetic resonance venography (CMRV) is the basic imaging method in the evaluation of venous sinuses, since it is a non-invasive and non-irradiating technique [10]. Time-of-flight (TOF) and phase-contrast
(PC) are the techniques used in CMRV [26]. CMRV is the most common method used in scientific research to evaluate venous variations [22]. Our study is one of the first studies performed with the PC technique using a 1.5 Tesla magnetic resonance imaging (MRI) machine.

Address for correspondence: Ass. Prof. E. Doğan, Muğla Sıtkı Koçman University Education and Research Hospital, Department of Radiology, 228 sok. Obam sitesi No:15 Kötekli/Menteşe, Muğla, Turkey, tel: +90 5066619794, fax: +90 2522123599, e-mail: dr_e_dogan@hotmail.com; emrahdogan@mu.edu.tr

This article is available in open access under Creative Common Attribution-Non-Commercial-No Derivatives 4.0 International (CC BY-NC-ND 4.0) license, allowing to download articles and share them with others as long as they credit the authors and the publisher, but without permission to change them in any way or use them commercially. 
According to literature, right chain vascular structures are remarkably dominant. Why is that? Transverse sinus (TS) and sigmoid sinus (SS) originate from the posterior plexus, jugular vein (JV) originates from the anterior cardinal vein (ACV). The correlation between these embryological structures can be determined by comparison of dominance [17]. Can the embryological mechanisms be explained by evaluating the correlation of vascular structures originating from different embryological buds? Is the embryological mechanism independent or interdependent?

Venography is a technique mostly used to detect thrombosis. Filling defects (FDs) are the main diagnostic finding in CMRV, but it can also be seen as physiological except for thrombosis [4, 22]. The percentage of these defects had been evaluated in previous studies [1, 2, 4]; however, the points where the defect is located on the TS are not specified. In which segments are FDs common? Which physical and physiological mechanisms can be associated with these FDs?

The aim of our study was to determine the ability of the PC-CMRV technique to detect cranial anatomy, variations, thrombosis, to reveal the deficits of the technique and to discuss the reasons for these deficits on a physics basis.

\section{MATERIALS AND METHODS}

\section{Patients}

Ethics committee approval was obtained for this study with document number 0945/7. Two hundred fourteen patients who had CMRV were chosen for the preliminary exam. Patients with a history of operation, thrombosis, ischaemic change, tumour, congenital anomalies, small vessel disease and demyelinating diseases were excluded from the study. All subjects were followed up for two years to exclude thrombosis.

Finally, 136 patients were included in the study: 50 males and 86 females; mean age ( \pm standard deviation [SD]), $48.7 \pm 16.3$ years, range: 18-93 years. Males' mean age $( \pm S D)$ was $47.6 \pm 12.9$ years; range between 19 and 86 years. Females' mean age $( \pm S D)$ was $50.6 \pm 13.7$ years; range between 18 and 93 years. All our patients were in the adult age group. CMRV and conventional MRI's (CMRI) of all the patients were evaluated by one experienced radiologist and one neuroradiologist and evaluated together again in case of a discrepancy.

\section{Imaging examinations}

A 1.5-tesla PHILIPS (The New Intera Nova, Philips medical system, Best, Netherlands) device was used for scanning. CMRV examinations were performed using the three dimensional (3D) PC technique without applying any saturation band. Maximum intensity projections (MIPs) were created at the MR operating console for the 3D CMRV dataset. The standard parameters that were used were as follows: field of view $=$ matrix $230 / 70=256 \times 256$, slice 160 , thickness $=1, \mathrm{Col}=1, \mathrm{TR} / \mathrm{TE}: 16 / 6.8$. The images were obtained with axial sections in 3D/FFE sequence. The last images were created with a velocity encoding (VENC) method by applying bipolar gradients sequentially along the cardinal directions $(x-, y-$ and $z-)$.

\section{Image analysis}

The images obtained with the picture archiving and communication system (PACS) were scanned in different projections for various veins in each patient. All veins were evaluated in raw images and 3D MIP images obtained by PC technique.

Parameters: dominances of TS, SS and JV were determined according to gender. Measurements were taken $1 \mathrm{~cm}$ from the torcular herophili for the TS, $1 \mathrm{~cm}$ from the TS junction for the SS, $1 \mathrm{~cm}$ from the SS junction for the JV $[6,20]$. If there was a difference greater than $1 / 5$ between the sinuses, the larger side was evaluated as dominant.

In addition, superior sagittal sinus (SSS), inferior sagittal sinus (ISS), straight sinus (StS), internal cerebral vein (ICV), Galen vein (GV), basal vein of Rosenthal (BVOR), occipital vein (OV), Labbe vein (LV) and Trolard vein (TrIV) were evaluated bilaterally. All vein's PC visualisation rates were compared with anatomical cadaveric studies and we found the real visualisation. The bilateral TS was divided into three equal parts (I: Inner part, II: Middle part, III: Outer part) and physiological FDs' percentages were calculated.

\section{Statistical analysis}

The obtained data were enrolled and tabulated using the Office Excel (Microsoft) data recording system. The data were analysed using statistical software (SPSS, IBM). All continuous variables were expressed as counts and averages were calculated (mean \pm SD). Percentages were calculated for qualitative values. Pearson and chi-square analysis were used for com- 
Table 1. Transverse sinus (TS), sigmoid sinus (SS), jugular vein (JV) dominances according to gender and side

\begin{tabular}{|c|c|c|c|c|c|c|}
\hline \multirow[t]{2}{*}{ SINUS } & \multicolumn{2}{|c|}{ R-dominant } & \multicolumn{2}{|c|}{ L-dominant } & \multicolumn{2}{|c|}{ C-dominant } \\
\hline & Number & Percentage & Number & Percentage & Number & Percentage \\
\hline \multicolumn{7}{|l|}{ TS } \\
\hline Female & 30 & $34.88 \%$ & 25 & $29.06 \%$ & 31 & $36.04 \%$ \\
\hline Male & 22 & $44.00 \%$ & 12 & $24.00 \%$ & 16 & $32.00 \%$ \\
\hline Total & 52 & $38.23 \%$ & 37 & $27.95 \%$ & 47 & $32.35 \%$ \\
\hline \multicolumn{7}{|l|}{ SS } \\
\hline Female & 31 & $36.04 \%$ & 25 & $29.07 \%$ & 30 & $34.88 \%$ \\
\hline Male & 23 & $46.00 \%$ & 13 & $26.00 \%$ & 14 & $28.00 \%$ \\
\hline Total & 54 & $39.70 \%$ & 38 & $27.95 \%$ & 44 & $32.35 \%$ \\
\hline \multicolumn{7}{|l|}{ JV } \\
\hline Female & 27 & $31.40 \%$ & 20 & $23.25 \%$ & 39 & $45.34 \%$ \\
\hline Male & 19 & $38.00 \%$ & 10 & $20.00 \%$ & 21 & $42.00 \%$ \\
\hline Total & 46 & $33.82 \%$ & 30 & $22.06 \%$ & 60 & $44.12 \%$ \\
\hline
\end{tabular}

parisons. The $p$ value $<0.05$ was accepted as statistically significant. Kendal Tau B test was performed in the non-parametric correlation analysis.

\section{RESULTS}

Dominances of TS, SS and JV were evaluated:

- for TS: Right (R)-dominance was found in 52 (38.23\%), left (L)-dominance in 37 (27.95\%), co-dominance in 47 (32.35\%) of the patients;

- for SS: R-dominance was found in 54 (39.70\%), L-dominance in 38 (27.95\%), co-dominance in 44 (32.35\%) of the patients;

- for JV: R-dominance was found in 46 (33.82\%), L-dominance in 30 (22.06\%), co-dominance in 60 $(44.12 \%)$ of the patients.

According to gender:

- for males; TS: R-dominance was found in 20 (44.00\%), L-dominance in 12 (24.00\%), co-dominance in 16 (32.00\%) of the patients; SS: R-dominance was found in $23(46.00 \%)$, L-dominance in $13(26.00 \%)$, co-dominance in $14(28.00 \%)$ of the patients; JV: R-dominance was found in 19 (38.00\%), L-dominance in 10 (20.00\%), co-dominance in 21 (42.00\%) of the patients;

- for females; TS: R-dominance was found in 30 (34.88\%), L-dominance in 25 (29.06\%), co-dominance in 31 (36.04\%); SS: R-dominance was found in 31 (36.04\%), L-dominance in 25 (29.07\%), co-dominance in 30 (34.88\%) of the patients; JV: R-dominant was found in 27 (31.40\%), L-dominance in 20 (23.25\%), co-dominance in 39 $(45.34 \%)$ of patients.
The TS, SS and JV's dominances' prevalence and frequency according to gender group are demonstrated in Table 1.

There was no statistically significant difference according to gender (TS: $p=0.567$; SS: $p=0.507$; JV: $p=0.726)$.

Kendal Tau B correlation analysis was applied to evaluate the relationship between TS, SS and JV dominance. The dominance relationship between the three evaluated sinuses on the right and left separately was statistically significant. The strongest correlation ( $\tau$ b: 0.945 ) was found between left TS and left SS. The lowest level of relationship ( $\tau \mathrm{b}$ : 0.791 ) was found between left TS and left JV. Tau B correlation between sinuses is demonstrated in Table 2.

All patients had SSS. However, SSS was completely visualised in 132 (97.06\%) patients. In $4(2.94 \%)$ patients, the anterior part of the SSS was not seen. ISS were visualised in 56 (41.17\%) of the patients (32 [37.21\%] females, 24 [48.00\%] males). StV, ICV and GV were visualised in all of the patients. BVOR was seen in 130 (95.59\%) and OC in $11(8.09 \%)$ of the patients. The percentages of detection of venous structures in the study are demonstrated in Table 3.

The existence of TrIV and LV were coded (as ' + ' present, '-' absent).

For TrlV: $\mathrm{R}+\mathrm{L}+$ was found in 28 (20.59\%), R+Lin 30 (22.06\%), R-L+ in 23 (16.91\%), R-L- in 55 (40.44\%) of the patients.

According to gender: 
Table 2. Dominance correlations of the transverse sinus (TS), sigmoid sinus (SS), jugular vein (JV) sinuses with Kendal Tau B test

\begin{tabular}{lccc}
\hline Dominancy & Compared sinuses & Tau-B correlation coefficient & Probability value \\
\hline Right dominant & ST and SS & $\tau_{\mathrm{b}}: 0.877$ & $\mathrm{p}: 0.000$ \\
& ST and VJ & $\tau_{\mathrm{b}}: 0.877$ & $\mathrm{p}: 0.000$ \\
Left dominant & ST and SS & $\tau_{\mathrm{b}}: 0.945$ & $\mathrm{p}: 0.000$ \\
& ST and VJ & $\tau_{\mathrm{b}}: 0.791$ & $\mathrm{p}: 0.000$ \\
Co-dominant & ST and SS & $\tau_{\mathrm{b}}: 0.836$ & $\mathrm{p}: 0.000$ \\
& ST and VJ & $\tau_{\mathrm{b}}: 0.836$ & $\mathrm{p}: 0.000$ \\
\hline
\end{tabular}

Table 3. Phase contrast visualisation ratio in cranial venous vessels

\begin{tabular}{|c|c|c|c|c|}
\hline \multirow[t]{2}{*}{ Anatomic localisation } & \multicolumn{2}{|c|}{ Present } & \multicolumn{2}{|c|}{ Absent } \\
\hline & Number & Percentage & Number & Percentage \\
\hline SSS: & 136 & $100 \%$ & 0 & $0 \%$ \\
\hline Complete & 132 & $97.06 \%$ & & \\
\hline Incomplete & 4 & $2.94 \%$ & & \\
\hline ISS: & 56 & $41.17 \%$ & 80 & $58.83 \%$ \\
\hline Female & 32 & $37.21 \%$ & 54 & $62.79 \%$ \\
\hline Male & 24 & $48.00 \%$ & 26 & $52.00 \%$ \\
\hline SV & 136 & $100 \%$ & 0 & 0 \\
\hline ICV & 136 & $100 \%$ & 0 & 0 \\
\hline GV & 136 & $100 \%$ & 0 & 0 \\
\hline BVOR & 130 & $95.59 \%$ & 6 & $4.41 \%$ \\
\hline OV & 11 & $8.09 \%$ & 125 & $91.91 \%$ \\
\hline
\end{tabular}

SSS — superior sagittal sinus; ISS — inferior sagittal sinus; SV — straight sinus vein; ICV — internal cerebral vein; GV — Galen vein; BVOR — basal vein of Rosenthal; OV — occipital vein

- for females, $R+L+$ was found in 17 (19.77\%), $\mathrm{R}+\mathrm{L}-$ in 19 (22.09\%), R-L+ in 13 (15.12\%), R-Lin $37(43.02 \%)$ of the patients;

- for males, $\mathrm{R}+\mathrm{L}+$ was found in $11(22.00 \%), \mathrm{R}+\mathrm{L}-$ in $11(22.00 \%), R-L+$ in 10 (20.00\%), R-L- in 18 (36.00\%) of the patients.

For LV: $\mathrm{R}+\mathrm{L}+$ was found in 73 (53.67\%), R+Lin 27 (19.85\%), R-L+ in 21 (15.44\%), R-L- in 15 $(11.03 \%)$ of the patients.

According to gender:

- for females, $R+L+$ was found in 49 (56.98\%), $\mathrm{R}+\mathrm{L}-$ in 17 (19.77\%), R-L+ in 10 (11.63\%), R-Lin $10(11.63 \%)$ of the patients;

- for males, $\mathrm{R}+\mathrm{L}+$ was found in $24(48.00 \%), \mathrm{R}+\mathrm{L}-$ in $10(20.00 \%), R-L+$ in $11(22.00 \%), R-L-$ in $5(10.00 \%)$ of the patients.

There was no statistical difference according to gender ( $p=0.153$ for TrIV and $p=0.060$ for LV). Bilaterally, TrIV and LV visualisation rates according to the gender are demonstrated in Table 4.
Finally, physiological FDs in the TS were evaluated. They were present in 37 of 136 patients (27.2\%). Ten of these patients had FDs in more than one segment. In total 47 FDs were determined. Thirty-four of them were in the middle segments.

Two (1.48\%) patients had FDs in the right outer part: one $(0.74 \%)$ partial and one $(0.74 \%)$ complete. Both of them were in the non-dominant sinuses. Fifteen $(11.03 \%)$ FDs were in the middle part of the right TS: 13 (9.55\%) were partial and $2(1.48 \%)$ complete. Thirteen were in the non-dominant sinuses, whereas two in the co-dominant sinuses. Two (1.48\%) patients had FDs in the right inner part: one $(0.74 \%)$ partial and one $(0.74 \%)$ complete. Both of them were in the non-dominant sinuses. Three $(2.21 \%)$ patients had FDs in the left inner part: two (1.48\%) partial and one $(0.74 \%)$ complete. Three of them were in non-dominant sinuses. Nineteen (13.97\%) were in the middle part of the left TS: 16 (11.76\%) partial and three $(2.21 \%)$ complete. Sixteen were in the non-dominant 
Table 4. Phase contrast visualisation ratio of Trolard and Labbe veins

\begin{tabular}{|c|c|c|c|c|c|c|c|c|}
\hline \multirow[t]{2}{*}{ Veins } & \multicolumn{2}{|c|}{$\mathrm{R}+\mathrm{L}+$} & \multicolumn{2}{|c|}{$\mathrm{R}+\mathrm{L}-$} & \multicolumn{2}{|c|}{ R-L+ } & \multicolumn{2}{|c|}{ R-L- } \\
\hline & Number & Percentage & Number & Percentage & Number & Percentage & Number & Percentage \\
\hline \multicolumn{9}{|l|}{ Trolard } \\
\hline Female & 17 & $19.77 \%$ & 19 & $22.09 \%$ & 13 & $15.12 \%$ & 37 & $43.02 \%$ \\
\hline Male & 11 & $22.00 \%$ & 11 & $22.00 \%$ & 10 & $20.00 \%$ & 18 & $36.00 \%$ \\
\hline Total & 28 & $20.59 \%$ & 30 & $22.06 \%$ & 23 & $16.91 \%$ & 55 & $40.44 \%$ \\
\hline \multicolumn{9}{|l|}{ Labbe } \\
\hline Female & 49 & $56.98 \%$ & 17 & $19.77 \%$ & 10 & $11.63 \%$ & 10 & $11.63 \%$ \\
\hline Male & 24 & $48.00 \%$ & 10 & $20.00 \%$ & 11 & $22.00 \%$ & 5 & $10.00 \%$ \\
\hline Total & 73 & $53.67 \%$ & 27 & $19.85 \%$ & 21 & $15.44 \%$ & 15 & $11.03 \%$ \\
\hline
\end{tabular}

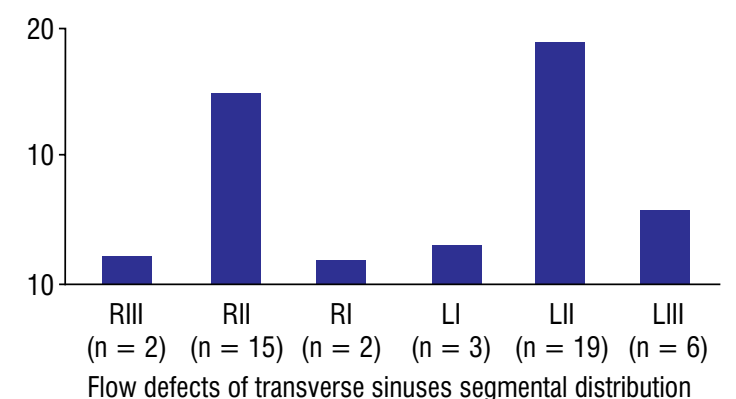

Figure 1. Segmental distribution of filling defects in transverse sinuses; $\mathrm{R}$ - right; $\mathrm{L}$ - left.

sinuses, whereas three in the co-dominant sinuses. Six $(4.41 \%)$ were in the outer part of the left TS: one $(0.74 \%)$ partial and five (3.67\%) complete. Five were in the non-dominant sinuses, whereas one in the co-dominant sinuses. There was no FD in the dominant sinuses. The FDs according to segments were demonstrated in Figure 1.

\section{DISCUSSION}

The dominance of the cerebral venous vessels is crucial before the radical neck dissection, excision of tumours invading the TS, SS, JV or glomus jugular tumours that may require ligation of the internal JVs [8]. In this study, TS, SS, and JV's dominances were found as right dominance (44\%), co-dominance (32\%) and left dominance (24\%) (Fig. 2). Although the percentages change, the order didn't change for both anatomic and radiological studies as well as this study $[4,5,16]$. Why right dominance is more visualized than left? We can find the answer to this question in the hypotheses belonging to the embryological development period. The superior vena cava originates from the right ACV together with the right

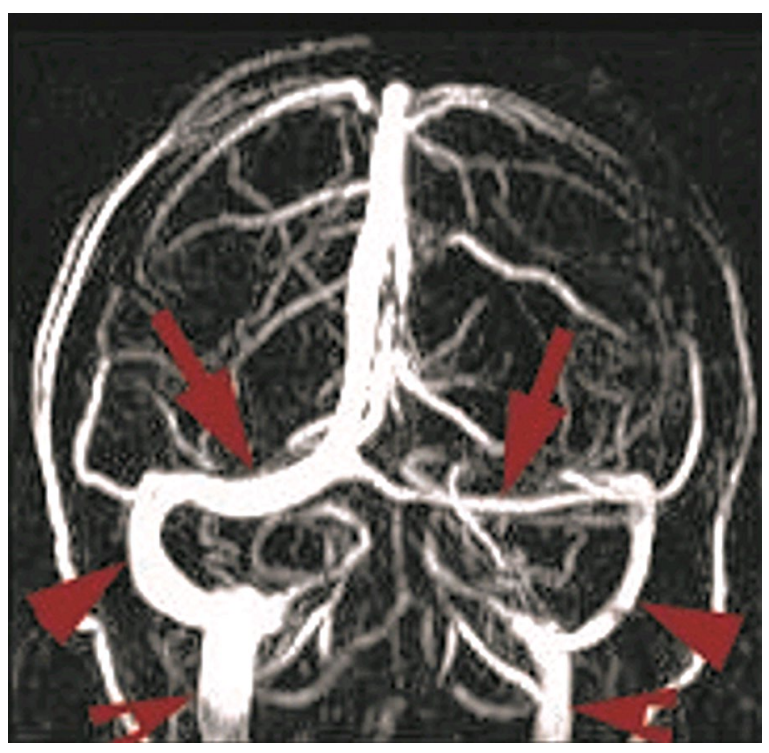

Figure 2. Venous sinuses are dominant in right (arrow: sigmoid sinus; arrowhead: transverse sinus; cut arrowhead: jugular vein).

JV. The caudal part of the left ACV largely regresses in the development process. If it does not regress, an anomaly called double vena cava occurs. ACVs merge with the posterior plexus which gives origin to TS and SS. Earlier joins coinciding with the regression process on the left, possibly lead to recessive left vessel chain. ACV regression on the left side influences not only JV but also TS and SS $[17,28]$. This study revealed that if the TS was dominant, the SS and JV were also dominant on the same side [26]. It showed that ipsilateral embryological buds move together. This information supports the hypothesis above.

Since the use of oral contraceptives and pregnancy is associated with cranial venous thrombosis, the use of CMRV is more common in women [22]. In our study, the majority of our patients were females 
(86 females, 50 males). There was no statistically significant gender-related difference between the TS, SS and JV dominances $(p=0.567$ for TS, $p=0.507$ for SS, $p=0.726$ for JV). The results of Goyal et al. [14] were similar.

Phase contrast's venous detection rates were evaluated and compared with anatomic studies. SSS was found at a rate of $100 \%$. This finding is similar to the literature $[1,2,4]$. SSS develops from the marginal sinus. Partial fusion defect at the attachment point of the marginal sinus to the foramen cecum causes a partial growth defect anteriorly. This situation is called partial split sinus (PSS) [2]. In Kaplan and Browder's cadaveric series, PSS prevalence was found as $6 \%$ [14]. In our study, PSS variant of the SSS was observed at a rate of $2.94 \%$. The signal loss may occur in the anterior section because the flow is going in the same direction as the artery, when an inferior saturation band is used to prevent arterial flow in TOF images [20]. In comparison with cadaveric studies, the percentage values are lower in our study. Its mean is that there is no signal loss due to PC technique in this area, unlike TOF.

In radiological studies, the presence of the ISS was noted between $33 \%$ and $43 \%[9,18]$. In our study, it was found at a rate of $41.17 \%$ (Fig. 3). In this case, it is necessary to look at the cadaveric studies. According to these ones, ISS was not detected in only $1 \%$ of the cases. To sum up, normally ISS is present but devices' visualisation and technique aren't adequate to show this vessel [13]. We looked at a study performed with more primitive device (0.35-tesla low-resolution) conducted by Sharma to explain more clearly the effect of device quality on venous visualisation. Visualisation rates with the low-tesla device of ISS, BVOR, ICV are respectively $11 \%, 34 \%, 60 \%$.

In this study, many vascular structures that we visualised could not be sufficiently imaged. We can say that the lower the quality of the device, the poorer the visualisation [23]. Visualisation of minor venous vessel with 3T, 5T and 7T devices probably will be higher than our study. It is open to further research.

In our study, BVOR was detected at a rate of $95.58 \%$. It was not observed unilaterally in five and bilaterally in one of the patients. In Ayanzen's TOF research, BVOR was detected in $91 \%$ of the patients. Our data are close to these findings [4]. We didn't find a cadaveric study to compare the percentage of basal vein [13]. StS, ICV and GV were detected in all of the patients. Results of other studies were similar to

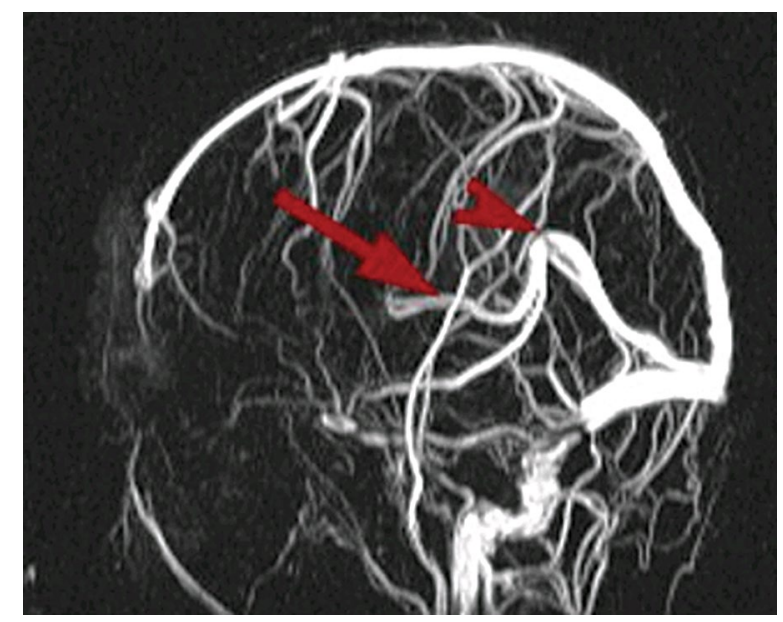

Figure 3. Internal cerebral veins (arrow) and Galen vein (arrowhead) in maximum intensity projections images.

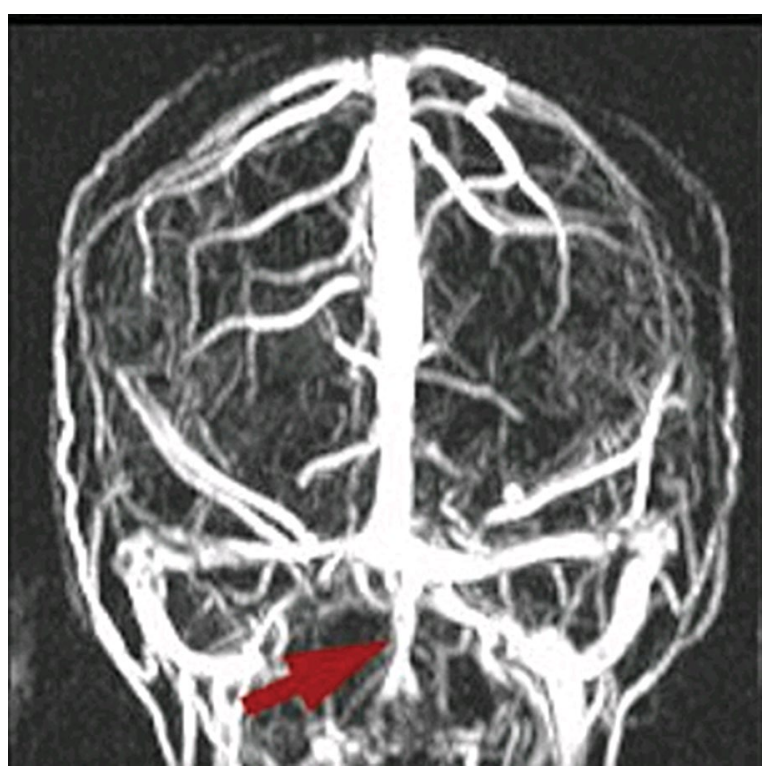

Figure 4. Occipital veins in posterior view of maximum intensity projections images (arrow).

ours $[4,23]$. When FDs are found in $100 \%$ visualised veins, it should be accepted primarily as pathological.

It has been reported that the OV is more prominent in patients with thrombosis [24]. If the TS and SS are hypoplastic, the OV is used as an alternative outflow pathway [20]. In our study, the OV detection rate with PC was found as $8.09 \%$. OV prevalence is between $4 \%$ and $35.5 \%$ according to data from reviewed anatomic and radiological TOF studies by Goyal et al. [11]. Our results were included in the aforementioned interval (Fig. 4). 


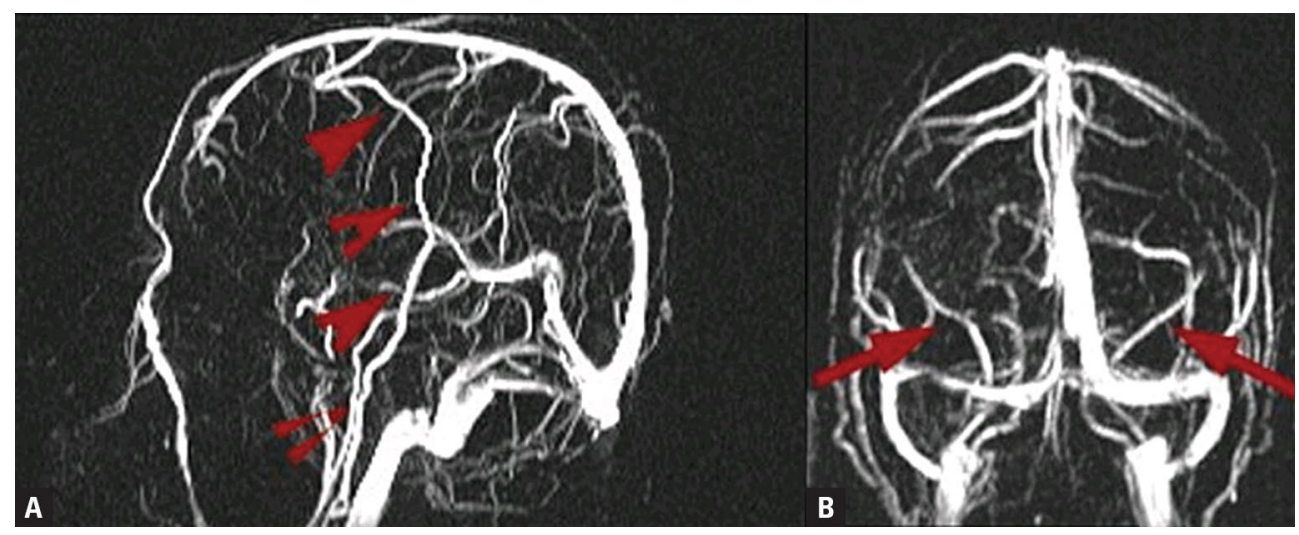

Figure 5. Trolard vein (arrowheads) and Labbe veins (arrows).

Trolard and Labbe veins form the main venous communicant anastomotic network. The LV provides the connection between the Sylvian veins and the posterior group, whereas the TrlV provides the connection between the SSS and the Sylvian veins. For this reason, it is also named as Trolard-Labbe circle. For example, when JV ligation is performed, if this network is not fully developed, venous infarct is probable. The LV should be preserved in temporal lobectomies and surgical interventions for epilepsy. Isolated thrombosis in LV, TrIV and related infarct cases have been reported in the literature [7, 22]. The angiographic studies elaborating information about these veins are present too [25]. Returning to our main subject, after we indicated why the LV and TrlV should be fully visualized and their clinical importance; our technique's detecting rate of LV and TrIV is close to other anatomical and radiological studies $[1,2,4]$. In addition, during our study, we noticed that there was no study according to gender regarding LV and TrIV, and we added it as a subtopic to the paper. There was no statistically significant difference ( $p=0.153$ for TrlV and $p=0.060$ for LV) according to gender (Fig. 5A, B).

Conventional MRI evaluation can give useful findings about thrombosis, but it is not adequate in the final diagnosis. In MRl; the intensity of the thrombus changes according to the period. What is the weaknesses of CMRI and CMRV? Thrombus due to effect of deoxyhaemoglobin appears isointense at $\mathrm{T} 1$ and hypointense at $\mathrm{T} 2$ in the first 5 days. During this period, the hypointensity of the thrombus at $\mathrm{T} 2$ makes it impossible to be detected in CMRI. Thus, venous thrombus can only be detected by angiography [22]. Since $7 \%$ of deaths due to venous thrombosis hap- pens in this period, it is important for the patient to be diagnosed early. Unfortunately, diagnosis is usually delayed for 7 days [20]. In these early days, where CMRI does not show any benefit and there is a risk of mortality, misleading sinus FDs are more important. Patients are mostly diagnosed within 5-15 days. This phase is also called the methaemoglobin phase. During this period, the thrombus T1 and T2 are hyperintense [22]. T1 hyperintensity is reflected as hyperintense in TOF technique also. Deleting of the flow void leads to interpreting the CMRV as normal. This situation is not seen in the PC technique. In this period PC is superior to TOF [20]. After 15 days, re-canalisation phase (chronic phase) starts [22]. In the chronic phase, pathways form within the thrombus. There is also dural enhancement accompanying capillary formation. Thus, it leads to a false negative result in contrast-enhanced MRI angiographic evaluation [20]. In this period, it cannot be mentioned that contrast enhanced MR angiography, which is accepted to be more advantageous than non-contrast CMRV, is superior to TOF and PC. It will be useful to evaluate CMRV FDs by comparing them with CMRI. Each technique has its pros and cons according to the period. It will be useful to evaluate CMRV images together with CMRI to prevent FDs from causing false diagnosis. In the early period, contrast enhanced MRI angiography, digital subtraction angiography in selected cases can be used for diagnosis [21].

Time-of-flight technique is used overwhelmingly in the CMRV examinations. The reason why PC research is rarely used is the long time according to TOF scanning [26]. Before mentioning the physical mechanisms of FDs, let's briefly talk about the general points of the techniques. Phase shift is undesirable in 


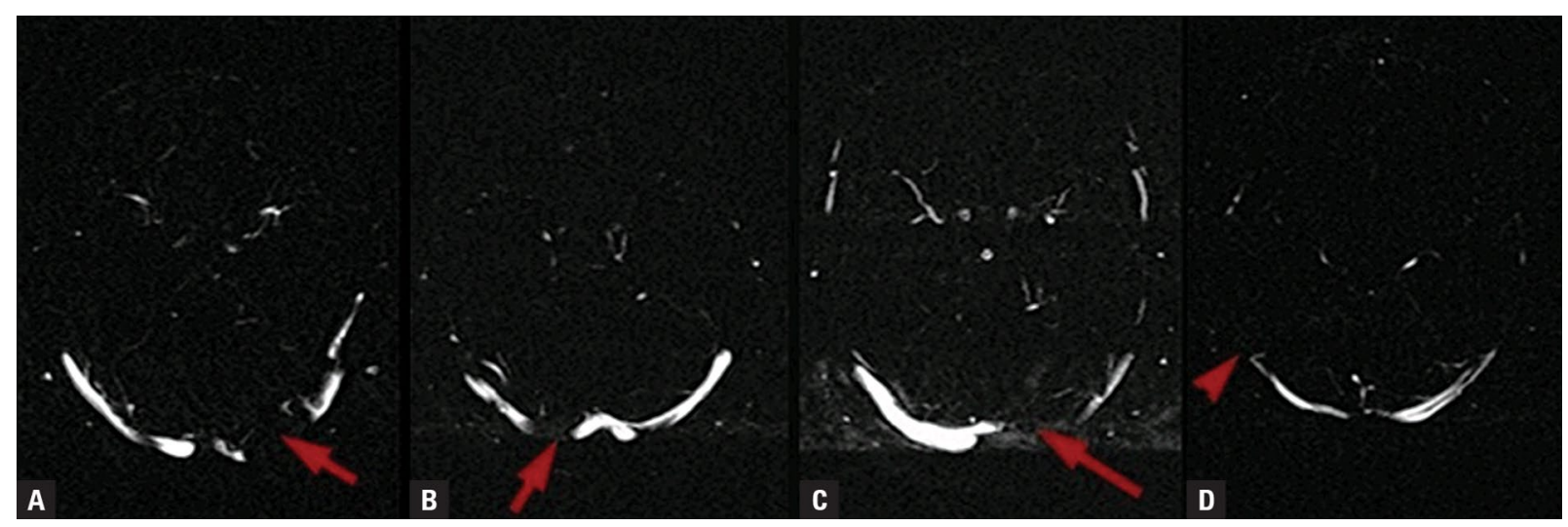

Figure 6. Physiological filling defects of transverse sinus; A. Filling defect of left inner and middle segments (arrow); B. Filling defects of right inner segment (arrow); C. Filling defect of left inner and middle segments (arrow); D. Filling defect of right outer segment (arrowhead).

TOF; therefore, "phase compensation technique" is used to prevent this. Phase shift that we want to prevent in TOF constitutes the basic of imaging in PC. In this technique, images are taken in pairs (phase shift) while operating in $(+)$ and $(-)$ gradient directions. Fixed textures are removed from the image with "image subtraction"; thus, only vascular structures are made visible. The technique is not sensitive to saturation due to flow; consequently, vascular structures with slow blood flow are better visualised in PC than in TOF. In addition, functional information such as flow direction and speed can be obtained with this technique [15]. PC's background suppression feature and anatomic detailing are superior to TOF's ones $[26,29]$.

Phase contrast has many disadvantages. The duration is long in PC and the eddy current effect is evident. It is sensitive to turbulence, spin saturation and intervoxel dephasing. It is also affected by intrinsic factors of the nucleus. Gradient imperfection secondary to inappropriate setting is creating aliasing artefacts in the flow direction as well. Gradient performance is directly related to device quality. There are many biomedical engineering articles in the literature about PC gradient settings. It is necessary to predict the appropriate gradient phase in advance [26].

Apart from this, there are some factors that cause FDs independent from the technique. This group consists of arachnoid granules (AG) and fibrotic bands located in the sinuses [20]. SSS and TSs are the most common places where AGs are seen. The majority of them are located between the middle and lateral parts in the TS $(92 \%)[3,12,27]$. Apart from the prominent eddy current effect due to PC technique, AG contributes to the formation of FD with mechanical effects
$[3,27]$. Fibrotic bands can make mechanical barrier effect too, but it is rarely seen [20].

The FD were detected in the centre (R2-L2) at the rate of $72.3 \%$, in the inner part (R3-L1) at the rate of $10.3 \%$, in the outer part (R1-L3) at the rate of $17 \%$ in TS (Fig. 6). All the disadvantages resulting from intrinsic nuclear factors mentioned above are present in all of the segments. Gradient effects are more pronounced on sharp turns in the segments R3-L1 and R1-L3 (outer and inner parts of TS). Besides, when the eddy current effect and the over mentioned physical barrier effects (AG) are added, a complex set of causes creates artefacts in corners and central segments $[19,26,29]$.

The movements of the spins in the presence of magnetic field gradients change the phase of the MR signal. These effects occur if blood flow goes a long way in the imaging volume, such as the TS. Phase shifts created by this movement cause artefacts in the phase coding direction and degrade image quality. This physical effect is more pronounced in PC than TOF [29]. The intrinsic nuclear factors contribute also to cause L2-R2 (middle parts of right and left TS) midpoint artefacts. Since the flow continues on a linear line, it will not be affected by the gradient effect's refraction. The AGs is considered as the primary flow defect factor in the middle part. AGs are concentrated between the middle and lateral segments. When blood crosses a physiological barrier like AG, the eddy current effect is towards the part where the blood is going, not where it comes from. Considering the anatomical point where AGs are concentrated, this area exactly corresponds to the middle part of the sinus [29]. Due to the fact that the venous sinuses 
are structures that don't have a muscularis mucosa that can expand according to the flow rate and they don't contain valves, two-way flow is possible and reverse flow is more pronounced in the middle section according to the hydro physics rules $[22,29]$. Add to this, the venous sinuses that are connected to the right atrium by a relatively short vascular way without valves, are affected by diastolic contractions [28]. Another question is why physiological FDs are not usually detected in the SSS in MRI? Two theorems can be put forward for this. The first is the flow rate. The TS is the main portal, collecting all venous blood. It is connected to the superior vena cava via the SS and JV. It is clear that SSS and other minor veins have lower flow rate. The second reason is the presence of AGs. Despite AGs are abundant in the SSS, giant AGs are found in the TS; thus, eddy current effects of giant AGs can be more prominent $[22,29]$.

The FDs were in the non-dominant or co-dominant TSs. No FD was found in the dominant TS. Since the non-dominant sinus percentage was higher on the left side, the frequency of FDs was higher on the left side too (right $40.4 \%$, left $59.6 \%$ ). To conclude, a FD in the dominant sinus should be primarily interpreted as thrombosis in PC studies. Physio pathologically, in recessive sinuses, eddy currents are sharper. When the diameter of the sinus is narrow, the number of spins per unit area decreases. The space-occupying effects of AGs become more pronounced.

The FD rates with TOF technique that were found by Ayanzen et al. [4], Alper et al. [2] and Ahmet et al. [1] are $31 \%, 24 \%$ and $10.8 \%$, respectively. In our study, the rate that was found is $27 \%$. We can say that our FD rates are similar to other TOF and PC studies.

The study has some limitations. The tests have not been confirmed with anatomic specimen.

\section{CONCLUSIONS}

This study is the first to assess the performance of PC technique by using normal anatomic and variations data. It is also the first time that dominances were correlated for explaining embryologic movements during the development of this region. Our results showed that the right vessel group was mostly dominant and found statistically significant correlations between the dominances of TS, SS and JV originating from different embryological buds. Left ACV regression also affects the posterior plexus during superior vena cava's embryological develop- ment at right. Our article supports this embryological theorem. The visualization degree of major veins in PC were sufficient but it was inadequate for determining some vessels like ISS (anatomic presence was approximately $100 \%$, visualisation was only $41.2 \%$ ). In comparison with our device, low-tesla device's visualisation of vessels is poorer. The higher the quality of the device, the higher the visualisation rate. FDs observed in the veins normally visualised should be primarily considered as thrombosis. For the first time, TS's FDs was evaluated according to segments in PC and causes of FD's were discussed based on physiopathology. Indeed, PC creates a high proportion of physiological FDs in TS. Results should not be trusted, especially if FDs are present in the middle part or non-dominant side. In the middle segment, defects are primarily related to slow flow and AG while eddy current artefacts affected the corner parts. TOF and PC are similar to create FDs. Let's remind, the research has been done in normal patients and concerns the PC's ability to demonstrate normal anatomy and its variations. PC may be superior for showing thrombosis because of the above mentioned physical rules. This topic will be the subject of future research.

\section{Declarations}

This is a retrospective study. Approval was obtained from izmir Atatürk Training and Research Hospital with 0945/7 before the study. The data were obtained from the PACS system and no illegal or prohibited data was included in the study.

During the study, the programmes purchased by Muğla Sıtkı Koçman University and İzmir Atatürk Education and research hospital were used legally.

\section{Conflict of interest: None declared}

\section{REFERENCES}

1. Ahmed MS, Imtiaz S, Shazlee MK, et al. Normal variations in cerebral venous anatomy and their potential pitfalls on 2D TOF MRV examination: Results from a private tertiary care hospital in Karachi. J Pak Med Assoc. 2018; 68(7): 1009-1013, indexed in Pubmed: 30317292.

2. Alper $F$, Kantarci $M$, Dane $S$, et al. Importance of anatomical asymmetries of transverse sinuses: an MR venographic study. Cerebrovasc Dis. 2004; 18(3): 236-239, doi: 10.1159/000079960, indexed in Pubmed: 15273441.

3. Apaydın F, Yalçınoğlu O, Yıldız A, et al. Arachnoid granulations in the transverse sinuses of a patient with ocular melanoma. J Clin Neurosci. 2003; 10(1): 132-134, doi: 10.1016/s0967-5868(02)00112-1. 
4. Ayanzen RH, Bird RC, Keller PJ, et al. Cerebral MR Venography: Normal Anatomy and Potential Diagnostic Pitfalls. Am J Neuroradiology. 2000; 21(1): 74-78, indexed in Pubmed: 10669228.

5. Browning $\mathrm{H}$. The confluence of dural venous sinuses. Am J Anat. 1953; 93(3): 307-329, doi: 10.1002/ aja.1000930302, indexed in Pubmed: 13104335.

6. Canedo-Antelo M, Baleato-González S, Mosqueira AJ, et al. Radiologic Clues to Cerebral Venous Thrombosis. Radiographics. 2019; 39(6): 1611-1628, doi: 10.1148/ rg.2019190015, indexed in Pubmed: 31589585.

7. Cullen S, Demengie F, Ozanne A, et al. The anastomotic venous circle of the base of the brain. Interv Neuroradiol. 2005; 11(4): 325-332, doi: 10.1177/159101990501100404, indexed in Pubmed: 20584444.

8. Durgun B, llglt ET, Cizmeli MO, et al. Evaluation by angiography of the lateral dominance of the drainage of the dural venous sinuses. Surg Radiol Anat. 1993; 15(2): 125-130, doi: 10.1007/BF01628311, indexed in Pubmed: 8367790.

9. Farb RI, Scott JN, Willinsky RA, et al. Intracranial venous system: gadolinium-enhanced three-dimensional MR venography with auto-triggered elliptic centric-ordered sequence-initial experience. Radiology. 2003; 226(1): 203-209, doi: 10.1148/radiol.2261020670, indexed in Pubmed: 12511691.

10. Ferro JM, Aguiar de Sousa D. Cerebral venous thrombosis: an update. Curr Neurol Neurosci Rep. 2019; 19(10): 74, doi: 10.1007/s11910-019-0988-x, indexed in Pubmed: 31440838.

11. Goyal G, Singh R, Bansal N, et al. Anatomical variations of cerebral MR venography: is gender matter? Neurointervention. 2016; 11(2): 92-98, doi: 10.5469/neuroint.2016.11.2.92, indexed in Pubmed: 27621945.

12. Haroun AA, Mahafza WS, Al Najar MS. Arachnoid granulations in the cerebral dural sinuses as demonstrated by contrast-enhanced 3D magnetic resonance venography. Surg Radiol Anat. 2007; 29(4): 323-328, doi: 10.1007/ s00276-007-0211-7, indexed in Pubmed: 17483869.

13. Ivashchuk RG, Tubbs $S$. Anatomy, imaging and surgery of intracranial dural venous sinuses, 1st ed. Elsevier Book, Seattle, Washington 2020: 29-35.

14. Kaplan HA, Browder J. Atresia of the rostral superior sagittal sinus: substitute parasagittal venous channels. J Neurosurg. 1973; 38(5): 602-607, doi: 10.3171/ jns.1973.38.5.0602, indexed in Pubmed: 4711634.

15. Konez $\mathrm{O}$. Manyetik rezonans görüntüleme [Turkish Book]. 1st Ed. Nobel tıp kitap evleri Itd, Istanbul 1995: 12-95.

16. Manara R, Mardari R, Ermani M, et al. Transverse dural sinuses: incidence of anatomical variants and flow artefacts with 2D time-of-flight MR venography at 1 Tesla. Radiol Med. 2010; 115(2): 326-338, doi: 10.1007/s11547-0100480-9, indexed in Pubmed: 20058094.

17. Manjila S, Bazil T, Thomas M, et al. A review of extraaxial developmental venous anomalies of the brain involv- ing dural venous flow or sinuses: persistent embryonic sinuses, sinus pericranii, venous varices or aneurysmal malformations, and enlarged emissary veins. Neurosurg Focus. 2018; 45(1): E9, doi: 10.3171/2018.5.FOCUS18107, indexed in Pubmed: 29961384.

18. Mattle HP, Wentz KU, Edelman RR, et al. Cerebral venography with MR. Radiology. 1991; 178(2): 453-458, doi: 10.1148/radiology.178.2.1987608, indexed in Pubmed: 1987608.

19. Ozsvath RR, Casey SO, Lustrin ES, et al. Cerebral venography: comparison of $\mathrm{CT}$ and MR projection venography. Am J Roentgenol. 1997; 169(6): 1699-1707, doi: 10.2214/ ajr.169.6.9393193, indexed in Pubmed: 9393193.

20. Provenzale JM, Kranz PG. Dural sinus thrombosis: sources of error in image interpretation. Am J Roentgenol. 2011; 196(1): 23-31, doi: 10.2214/AJR.10.5323, indexed in Pubmed: 21178043.

21. Rizzo L, Crasto SG, Rudà R, et al. Cerebral venous thrombosis: role of $\mathrm{CT}, \mathrm{MRI}$ and MRA in the emergency setting. Radiol Med. 2010; 115(2): 313-325, doi: 10.1007/s11547010-0493-4, indexed in Pubmed: 20091136.

22. Sajjad ZM. and MRV in cerebral venous thrombosis. J Pak Med Assoc. 2006; 56(11): 523-526, indexed in Pubmed: 17183982

23. Sharma UK, Sharma K. Intracranial MR venography using I ow-Field magnet: normal anatomy and variations in nepalese population. J Nepal Med Assoc. 2012; 52(186): 61-65, doi: 10.31729/jnma.3.

24. Shin HS, Choi DS, Baek HJ, et al. The oblique occipital sinus: anatomical study using bone subtraction 3D CT venography. Surg Radiol Anat. 2017; 39(6): 619-628, doi: 10.1007/s00276-016-1767-x, indexed in Pubmed: 27796494.

25. Silva PS, Vilarinho A, Carvalho B, et al. Anatomical variations of the vein of Labbé: an angiographic study. Surg Radiol Anat. 2014; 36(8): 769-773, doi: 10.1007/s00276014-1264-z, indexed in Pubmed: 24531417.

26. Surendrababu N, Livingstone RS. Variations in the cerebral venous anatomy and pitfalls in the diagnosis of cerebral venous sinus thrombosis: Low field MR experience. Indian J Med Sci. 2006; 60(4): 135-142, doi: 10.4103/00195359.24677.

27. Trimble CR, Harnsberger HR, Castillo M, et al. "Giant" arachnoid granulations just like CSF?: NOT!! Am J Neuroradiol. 2010; 31(9): 1724-1728, doi: 10.3174/ajnr.A2157, indexed in Pubmed: 20581064.

28. Tubbs RS, Goren O, McBain L. Anatomy, Imaging and Surgery of the Intracranial Dural Venous Sinuses, 1 st Ed. Elsevier Book, Seattle, Washington 2020: 1-7.

29. Wang J, Wang J, Sun J, et al. Evaluation of the anatomy and variants of internal cerebral veins with phase-sensitive MR imaging. Surg Radiol Anat. 2010; 32(7): 669-674, doi: 10.1007/s00276-010-0669-6, indexed in Pubmed: 20422190 . 